\title{
47,XXY/48,XXXY/49,XXXXY mosaic with hydrocephaly: a case report and review of the literature
} Jesús E Dueñas-Arias ${ }^{\dagger 1}$, Maribel Aguilar-Medina ${ }^{\dagger 2}$, Eliakym ArámbulaMeraz $^{2}$, Juliana B Valenzuela-Camacho', Angelina Vega-Solano ${ }^{1}$, Julio Granados ${ }^{3}$ and Rosalío Ramos-Payán*2

Address: ${ }^{1}$ Departamento de Genética, Hospital Pediátrico de Sinaloa. Culiacán, Sinaloa, México, ${ }^{2}$ Laboratorio de Inmunología y Biología Molecular, Doctorado en Biotecnología, Facultad de Ciencias Químico Biológicas, Universidad Autónoma de Sinaloa. Culiacán, Sinaloa, México and ${ }^{3}$ Departamento de Inmunología y Reumatología, Instituto Nacional de Ciencias Médicas y Nutrición Salvador Zubirán, México D.F., México

Email: Jesús E Dueñas-Arias - jedanet@yahoo.com; Maribel Aguilar-Medina - maribela2@excite.com; Eliakym ArámbulaMeraz - eliakymarambula@ hotmail.com; Juliana B Valenzuela-Camacho - juliana_70@yahoo.com; Angelina Vega-

Solano - angyqfb@hotmail.com; Julio Granados - julgrate@yahoo.com.mx; Rosalío Ramos-Payán* - ramospayan@yahoo.com.mx

* Corresponding author †Equal contributors

Published: 19 September 2007

Journal of Medical Case Reports 2007, I:94 doi:10.1186/1752-1947-1-94

Received: 29 May 2007

This article is available from: http://www.jmedicalcasereports.com/content/I/I/94

(c) 2007 Dueñas-Arias et al; licensee BioMed Central Ltd.

This is an Open Access article distributed under the terms of the Creative Commons Attribution License (http://creativecommons.org/licenses/by/2.0), which permits unrestricted use, distribution, and reproduction in any medium, provided the original work is properly cited.

\section{Background}

Klinefelter's syndrome is a common sex chromosomal abnormality observed in humans, with a prevalence of 1 in 500 males [1-3]. The clinical features are variable but often include infertility, gynecomastia, eunuchoidism, small testes and penis and hypergonadotropic hypogonadism. The syndrome is usually caused by the presence of one additional X chromosome (47,XXY aneuploidy), however, rare syndrome variants with $\mathrm{X}$ and $\mathrm{Y}$ polysomy, mosaicisms and aberrant chromosomes have been reported, including 46,XX, 48,XXXY, 48,XXYY, 49XXXXY, $47, \mathrm{XXY} / 48, \mathrm{XXXY}$ and $48, \mathrm{XXXY} / 49, \mathrm{XXXXY}$ among others [4].

The 49,XXXXY chromosomal constitution was described by Fraccaro in 1960 [5]. Despite being usually considered as a Klinefelter variant, the $49, \mathrm{XXXXY}$ aneuploidy shows a distinct phenotype and more severe clinical features $[6,7]$. More than one hundred cases have been reported, with a frequency of 1 in 85,000 males $[1,8]$.

Klinefelter with mosaicisms presents a moderate phenotype and accounts for $15 \%$ of all cases [6]. The $47, \mathrm{XXY} /$ $48, \mathrm{XXXY} / 49, \mathrm{XXXXY}$ is a very rare mosaic, and to our knowledge, only three cases have been reported until now [9-11]. In this paper, we described XXY/XXXY/XXXXY mosaicism in a newborn with congenital obstructive hydrocephalus and mild cardiopathy.

\section{Case presentation}

Clinical history. A 5-day old male was admitted at the Pediatric Hospital of Sinaloa in México. His parents were 
unrelated. He was born by cesarean section at week 39 due to fetal arrhythmias and intrauterine growth retardation. He had an Apgar score of 8 at 1 minute and 9 at 5 minutes, $2 \mathrm{Kg}$ birth weight, $47 \mathrm{~cm}$ body length and $37 \mathrm{~cm}$ cephalic circumference. The patient was hospitalized because clinical examination revealed several congenital abnormalities: hypotrophy, macrocephaly, facial asymmetry, hypertelorism, low nasal bridge, low-set ears, micrognathia, short neck, narrow thorax, bulky abdomen, clinodactyly of the fourth and fifth fingers of both hands, hyperpigmented genitals, micropenis, hypospadia and a mid-scrotal septum with left cryptorchism.

Laboratory data. Radiographs showed no osseous abnormalities in joints and long bones. Echocardiography revealed a $1.6 \mathrm{~mm}$ arterial vessel with bidirectional flux and atrioventricular concordance. Abdominal ultrasound was normal, but anomalies were observed in the transfontanelle ultrasonography. Magnetic resonance imaging of the head confirmed the presence of supratentorial noncommunicating hydrocephaly with slightly hypoplastic corpus callosum (figure 1).

Genetic analysis. Cytogenetic study by GTG banding of peripheral lymphocytes showed a XXY/XXXY/XXXXY mosaicism (data not shown). FISH analysis of 207 cellular nuclei, using probes for chromosomes $\mathrm{X}$ and $\mathrm{Y}$, indicated a line proportion of $3 \%$ for $47, \mathrm{XXY}, 36 \%$ for $48, \mathrm{XXXY}$ and $61 \%$ for $49, \mathrm{XXXXY}$ (figure 2 ).

\section{Discussion}

Fraccaro's syndrome is caused by 49,XXXXY chromosomal aneuploidy and is a rare condition often classified as a Klinefelter's syndrome variant, however, as stated by
Peet and Hou, we propose that it should be diagnosed as an independent clinical syndrome $[8,7]$.

In this paper, we describe a newborn with a XXY/XXXY/ XXXXY mosaicism with Fraccaro's syndrome phenotype. The percentage of the $49, \mathrm{XXXXY}$ line was $61 \%$ as determined by nuclei FISH analysis (figure 1), which is in accordance with the clinical features of the patient. To our knowledge, this is the fourth report of this mosaicism in the literature, but with different line proportion and clinical phenotypes. Moreover, the child had mild cardiopathy and supratentorial non-communicating hydrocephalus (figure 2). Whether this congenital hydrocephaly is a new syndrome variant or just an independent event remains to be determined. As the presence of hydrocephaly in patients with XXY/XXXY/XXXXY mosaicism has not been reported before in any Klinefelter variants nor in Fraccaro's syndrome, it should be considered in future cases, during cytogenetic analysis and in the first six months after birth.

\section{Competing interests}

The author(s) declare that they have no competing interests.

\section{Authors' contributions}

DAJE provided genetic counselling to the parents. AMM and DAJE collected the data relative to this case report. VMJB and VSA conducted the data analysis and interpreted experiments and revised the manuscript. AMM, AME, GJ and RPR performed genetic studies and elaboration and drafting of the manuscript. All authors read and approved the final manuscript.
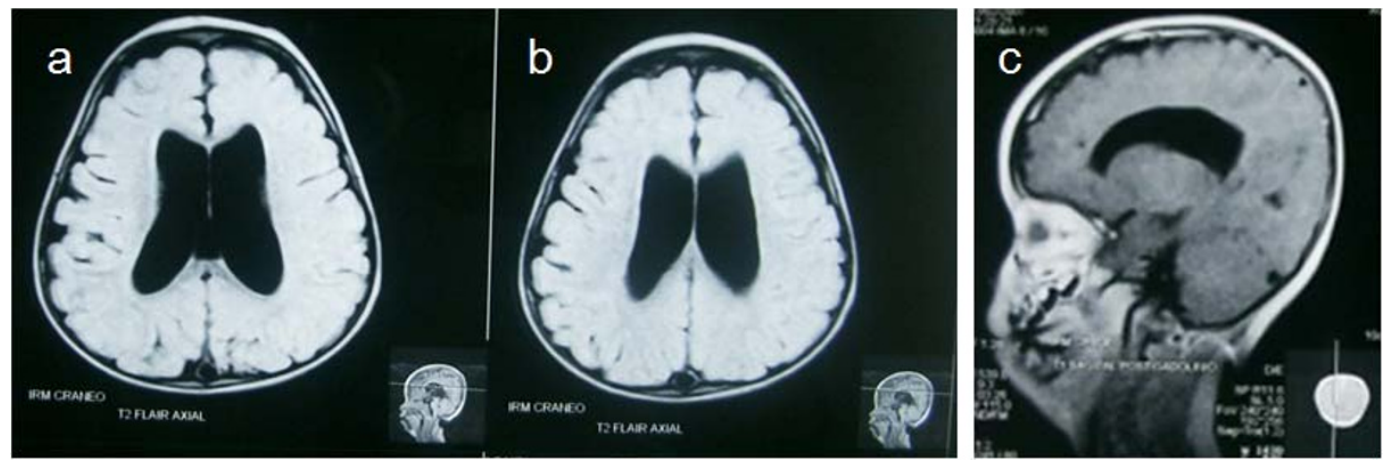

\section{Figure I}

Magnetic Resonance of head (MR-Head). MR-Head showing supratentorial non-communicating hydrocephalus (a, b) and slightly hypoplastic corpus callosum (c). 

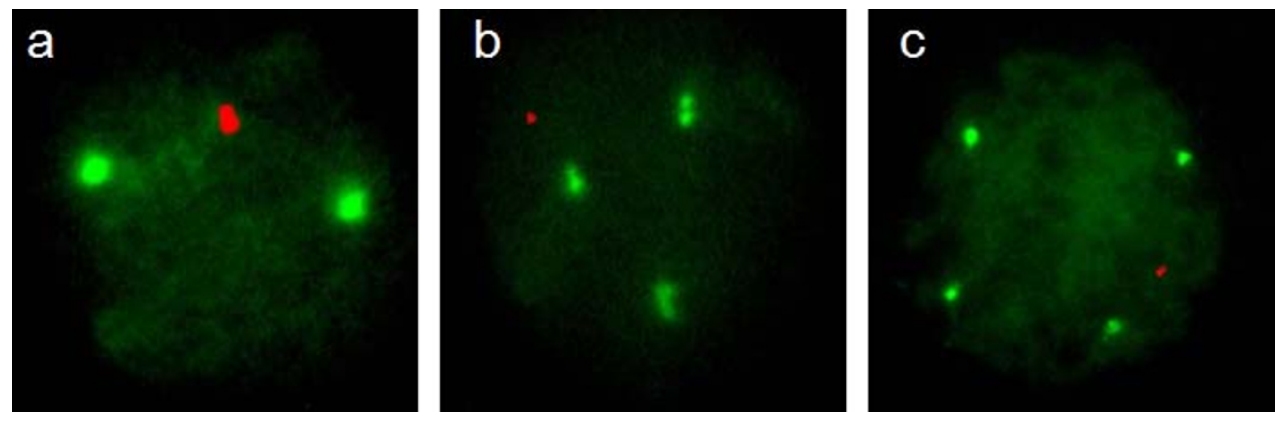

Figure 2

Fluorescence In situ Hybridization (FISH) assay. Nuclei FISH analysis of peripheral lymphocytes reveals a $X X Y / X X X Y /$ $X X X X Y$ mosaic in $a, b$ and $c$ respectively.

\section{Consent}

Written consent was obtained from the newborn's mother for publication of this case report.

\section{Acknowledgements}

Aguilar-Medina M, Ramos-Payán R, Arámbula-Meraz E are SNI fellows. This work was supported by the General Coordination of Research and Posgraduate (CGIP-PROFAPI) Universidad Autónoma de Sinaloa and CECYT (State Council of Science and Technology) from Sinaloa, México.

\section{References}

I. Visootsak J, Graham JM Jr: Klinefelter syndrome and other sex chromosomal aneuploidies. Orphanet J Rare Dis 2006, 24:42.

2. Jacobs PA, Strong JA: A case of human intersexuality having possible XXY sex-determining mechanism. Nature 1959, 2:164-167.

3. Klinefelter HF, Reifenstein EC, Albright F: Syndrome characterized by gynecomastia aspermatogenes without A-Leydigism and increased excretion of follicle stimulating hormone. J Clin Endocrinol Metab 1942, 2:6I5-627.

4. Hirschhorn K, Hirschhorn R, Fraccaro M, Book JA: Incidence of familial hyperlipemia. Science 1959, I29:716-7.

5. Fraccaro M, Kaijser K, Lindsten J: A child with 49 chromosomes. Lancet 1960, 2:899-902.

6. Wattendorf DJ, Muenke M: Klinefelter syndrome. Am Fam Physician 2005, 72:2259-62.

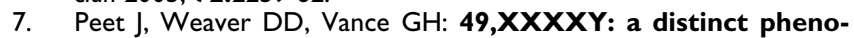
type. Three new cases and review. J Med Genet 1998, 35:420-4.

8. Hou JW: 49, XXXXY syndrome. Chang Gung Med J 2004, 27:55 I-4.

9. Tumba $A$ : Case of triple chromosome mosaicism XXY/ $\mathbf{X X X Y / X X X X Y . ~ U n i o n ~ M e d ~ C a n ~ 1 9 7 5 , ~ 1 0 4 : 7 3 9 - 4 4 . ~}$

10. Kardon NB, Beratis NG, Hsu LY, Moloshok RE, Hirschhorn K: $47, X X Y-48, X X X Y-49, X X X X Y$ mosaicism in a 4-year-old child. Am J Dis Child 197I, I 22:I60-2.

II. Guli E, Cellesi C: Klinefelter's syndrome with $\mathbf{X X X Y - X X Y -}$ XXXXY mosaic. Atti Accad Fisiocrit Siena 1967, I6:|28|-91.
Publish with Biomed Central and every scientist can read your work free of charge

"BioMed Central will be the most significant development for disseminating the results of biomedical research in our lifetime. "

Sir Paul Nurse, Cancer Research UK

Your research papers will be:

- available free of charge to the entire biomedical community

- peer reviewed and published immediately upon acceptance

- cited in PubMed and archived on PubMed Central

- yours - you keep the copyright
BioMedcentral 